\title{
Instantáneas
}

\section{Suplementos de vitaminas C y E y mortalidad por cáncer colorrectal}

Las vitaminas C y E son importantes antioxidantes que pueden reducir el riesgo de cáncer. Si los suplementos de estas vitaminas redujeran sustancialmente el riesgo de cáncer colorrectal (CCR), sus implicaciones para la salud pública serían importantes, dado que los suplementos vitamínicos son baratos y fáciles de administrar y que el CCR constituye la tercera causa más frecuente de muerte por cáncer en los hombres y mujeres de los Estados Unidos de América (EE.UU.). Solo se han realizado tres estudios prospectivos sobre la asociación entre el CCR y el uso de suplementos de vitaminas C o E y algunos de sus resultados indican una reducción del riesgo, particularmente con los suplementos de vitamina E. Sin embargo, ninguno de ellos ha analizado los resultados en función de la duración del consumo de los suplementos vitamínicos. Esto llevó a un grupo de investigadores de la American Cancer Society a analizar la asociación entre la mortalidad por CCR y el uso suplementos de vitamina C o E de forma aislada (no como complejos polivitamínicos) en una gran cohorte de adultos de los EE.UU.

Los individuos analizados formaban parte de la cohorte de 508351 varones y 676306 mujeres participantes en el Estudio de Prevención del Cáncer-II (CPS-II), todos ellos con más de 30 años de edad en 1982, momento de su inclusión en el estudio (promedio de 57 años para los varones y 56 para las mujeres).

Se determinó que hasta el 31 de diciembre de 1996 habían muerto 237452 (20,0\%), 944313 seguían vivos $(79,7 \%)$ y $2892(0,2 \%)$ habían dejado de ser seguidos por datos insuficientes. Se obtuvieron los certificados de defunción de 98,6\% de los fallecidos. Las causas de muerte se definieron de acuerdo con la novena edición de la Clasificación Internacional de Enfermedades. Se excluyeron 82349 individuos con antecedentes de cáncer, 158180 que no proporcionaron información suficiente sobre la frecuencia y duración del uso de las cuatro vitaminas (A, C, E y polivitaminas) que figuraban en el cuestionario, 110684 que tomaban uno o más de los suplementos vitamínicos menos de una vez a la semana, 17628 con la misma frecuencia y duración de uso de los cuatro suplementos vitamínicos, quienes podrían estar informando sobre su consumo de suplementos polivitamínicos y no de vitaminas individuales, y 103925 que no proporcionaron información sobre la dieta, la educación o el índice de masa corporal. Se analizaron los 711891 individuos restantes (334 125 hombres y 377766 mujeres).

A través del cuestionario realizado en 1982 se determinó la frecuencia y duración del consumo de los cuatro suplementos vitamínicos. Se consideraron consumidores diarios los individuos que decían tomar una vitamina en particular al menos 25 días al mes.

La asociación entre el consumo de suplementos de vitaminas C y E y el CCR se analizó con el modelo de riesgos proporcionales de Cox, teniendo en cuenta otros posibles factores de riesgo (edad, nivel educacional, índice de masa corporal, consumo de aspirina, tratamiento de reposición de estrógenos y consumo de vegetales y granos con alto contenido de fibra).

Durante los 14 años de observación (19821996) hubo 4404 muertes por CCR (2 468 en hombres y 1936 en mujeres). En términos generales, no se obtuvo ninguna prueba de que el consumo diario de suplementos de vitaminas $\mathrm{C}$ o $\mathrm{E}$, aunque fuera prolongado, se asociara con una reducción de la mortalidad por CCR. Las razones de las tasas (RR) fueron de 0,89 (intervalo de confianza de 95\%; IC95\%: 0,73-1,09) con el consumo a largo plazo (10 o más años) de vitamina C y de 1,08 (IC95\%: 0,85-1,38) con el consumo a largo plazo de vitamina $\mathrm{E}$.

En los análisis de subgrupos, el consumo diario de vitamina $\mathrm{C}$ a largo plazo se asoció con una tendencia a la disminución del riesgo en los varones ( $R R=0,75$; IC95\%: 0,57-1,00), pero no en las mujeres $(R R=1,05$; IC95\%: 0,80-1,38). El consumo a largo plazo de vitamina $\mathrm{C}$ también se asoció con una gran reducción del riesgo de muerte por CCR en los menores de 65 años (RR = 0,48; IC95\%: $0,28-0,81)$, pero no en los mayores de 65 años ( $R R=1,02 ;$ IC95\%: 0,83-1,26), y a una reducción del riesgo de muerte por cáncer rectal a todas las edades ( $R R=0,40$; IC95\%: 0,20-0,80), aunque no del riesgo de muerte por cáncer de colon $(\mathrm{RR}=0,98$; IC95\%: 0,80-1,20). El consumo a largo plazo de vitamina E presentó una tendencia no significativa a disminuir la mortalidad por CCR en los fumadores ( $R R=0,60$; IC95\%: 0,31-1,16), pero no en los no fumadores ni en los exfumadores. No se observaron interacciones importantes entre el consumo diario de vitamina $\mathrm{C}$ y de vitamina $\mathrm{E}$, independiente- 
mente de que se incluyeran o excluyeran los usuarios de polivitaminas.

Los resultados negativos de este estudio son importantes, dado que algunos estudios recientes han detectado un importante efecto protector de los suplementos de estas vitaminas antioxidantes frente al CCR. En uno de ellos, el consumo de suplementos de vitamina E se asoció con una disminución de $50 \%$ en el riesgo de CCR en mujeres.

Los resultados de los subanálisis deben ser interpretados con cautela. No hay otros estudios que indiquen que la reducción del riesgo con la vitamina $C$ sea mayor en los hombres que en las mujeres ni en los menores de 65 años que en los mayores, ni hay motivos para esperar que la vitamina $C$ tenga un efecto específico sobre el cáncer rectal, más que sobre el cáncer de colon.

Después de analizar las posibles explicaciones para la discrepancia entre estos resultados y los de estudios anteriores, los autores concluyen que el consumo a largo plazo de vitaminas $\mathrm{C}$ o E no parece tener un sustancial efecto protector frente a la mortalidad por CCR, aunque no se pueden excluir efectos beneficiosos en poblaciones malnutridas o en subgrupos específicos de la población. (Jacobs EJ, Connell CJ, Patel AV, Chao A, Rodriguez C, Seymour J, et al. Vitamin $C$ and vitamin $E$ supplement use and colorectal cancer mortality in a large American Cancer Society cohort. Cancer Epidemiol Biomarkers Prev 2001;10:17-23).

\section{Los problemas de salud de las personas sin hogar}

La falta de hogar tiene importantes implicaciones en la salud. Las personas sin hogar tienen mayor morbilidad y mortalidad que el resto de la población y se encuentran con importantes barreras en su acceso a la atención sanitaria. En este artículo se definen las características demográficas de este sector de la población, sus problemas de salud más frecuentes y la respuesta del sistema de salud a sus necesidades. Aunque los datos expuestos se refieren sobre todo a Canadá, buena parte de ellos son aplicables también a otros países, tanto desarrollados como en vías de desarrollo. En este artículo se consideran personas sin hogar tanto las que duermen en la calle, en vehículos, en edificios abandonados o en otros lugares no destinados a la habitación, como las que disponen de un refugio que no cumple los requisitos básicos de seguridad y salubridad.

Con base en la capacidad de los refugios para las personas sin hogar, se estima que en las nueve áreas metropolitanas más importantes de Canadá hay unas 8000 personas que duermen en estos refugios cada noche, lo cual representa un 5/10 000 de la población. El número de individuos que usan estos refugios al menos una vez al año es cinco veces mayor. Esta estimación no incluye a los individuos que duermen en la calle, más numerosos que los que duermen en refugios, y que, sólo en Vancouver, se estima que representan un 3/10 000 de la población. Estas cifras indican que el número total de personas sin hogar en Canadá es del orden de decenas de miles.

Las características demográficas de la población sin hogar varían de una ciudad a otra. Los varones solteros constituyen el grupo más numeroso en la mayoría de las ciudades canadienses: cerca de $70 \%$ en Vancouver, Edmonton y Calgary, y cerca de $50 \%$ en Ottawa. Generalmente son jóvenes, la mayoría entre 25 y 44 años de edad. Las mujeres solteras representan aproximadamente un cuarto de la población sin hogar en Vancouver, Edmonton y Toronto, pero solo 10\% en Calgary y Ottawa. Estudios realizados en los Estados Unidos de América (EE.UU.) indican que, en comparación con las mujeres sin hogar y con hijos, las mujeres solas tienden a ser mayores y a presentar antecedentes de enfermedad mental o abuso de drogas. La falta de hogar afecta también a muchas familias con hijos. Los jóvenes de menos de 20 a 25 años sin hogar suelen proceder de familias en las que han sufrido maltratos físicos o abusos sexuales. Este segmento está subestimado porque la mayoría de ellos no duermen en refugios. En Canadá, la falta de hogar afecta particularmente a los aborígenes. Por ejemplo, en Edmonton representan $35 \%$ de la población sin hogar, pero solo 3,8\% de la población total. Generalmente, los aborígenes se encuentran entre los que duermen en la calle, y no en refugios.

Las personas sin hogar tienen mayor riesgo de morir que el resto de la población. En comparación con la población joven de Québec, la tasa de mortalidad de los jóvenes sin hogar es 9 veces mayor para los varones y 31 veces mayor para las mujeres. En los EE.UU. la tasa de mortalidad de los varones sin hogar duplica a la de Canadá, diferencia que se debe a distintos factores, como las menores tasas de homicidio y de infección por el virus de la inmunodeficiencia humana (VIH), y, posiblemente, la universalidad del seguro de salud en Canadá.

Las personas sin hogar sufren una gran variedad de problemas médicos y sus enfermedades pueden ser mucho más graves debido a factores como la pobreza extrema, el retraso en la búsqueda de atención médica, el incumplimiento del tratamiento, el deterioro cognitivo y los efectos adversos sobre la salud de la falta de hogar en sí misma. Los que viven en la calle tienden a presentar peor estado de salud que los que residen en refugios.

Entre los problemas médicos más frecuentes en la población adulta sin hogar se encuentran las 
convulsiones, la neumopatía obstructiva crónica, la artritis y otras enfermedades musculoesqueléticas. Enfermedades como la hipertensión, la diabetes y la anemia suelen estar mal controladas. Las infecciones respiratorias son frecuentes y la higiene bucodental suele ser mala. Los problemas cutáneos y podiátricos también son frecuentes. Los que viven en la calle son particularmente propensos a la celulitis, impétigo, estasis venoso, escabiosis y pediculosis. El uso de calzado inadecuado, la exposición prolongada a la humedad, los largos períodos caminando y de pie y los traumatismos menores repetitivos conducen frecuentemente a la onicomicosis, tinea pedis, callos y pie de inmersión.

También hay un aumento del riesgo de tuberculosis, diagnóstico que debería ser tenido en cuenta en todo individuo sin hogar con fiebre y tos productiva persistente. Más de la mitad de los casos son primoinfecciones. Entre los sin hogar de Toronto, la incidencia de tuberculosis activa $(71 / 10000)$ es unas 10 veces superior a la media de la provincia de Ontario. El tratamiento de la tuberculosis activa puede verse dificultado por su incumplimiento, la infecciosidad prolongada y la resistencia a los fármacos. El tratamiento observado directamente proporciona mayores tasas de curación y menos recidivas. Las personas sin hogar con tuberculina positiva y sin tuberculosis activa son candidatas a la profilaxis observada directamente.

Los factores de riesgo de infección por VIH más frecuentes entre los jóvenes canadienses sin hogar son la prostitución, la multiplicidad de parejas sexuales, la falta de utilización de preservativos y el consumo de drogas inyectables. Las tasas de infección son muy variables entre ciudades, e incluso dentro de una misma ciudad (entre 2,2 y 11,3\% en Vancouver en 1988). El patrón de factores de riesgo de los adultos es diferente, con predominio del consumo de drogas sobre los comportamientos sexuales. La salud sexual y reproductiva constituye un importante problema para los jóvenes sin hogar. En Montreal, 25\% de ellos se han prostituido. Las enfermedades de transmisión sexual son muy frecuentes, incluso entre los que no se prostituyen; las más prevalentes son la gonorrea y la infección por Chlamydia.

La violencia es una amenaza constante para las personas sin hogar. Una encuesta realizada en Toronto reveló que, en el año anterior, $40 \%$ de los individuos sin hogar habían sido agredidos y $21 \%$ de las mujeres habían sido violadas. Los varones sin hogar tienen una probabilidad nueve veces mayor de ser asesinados, en comparación con los varones en general. Las lesiones involuntarias constituyen una de las principales causas de morbilidad y mortalidad, especialmente entre los varones; a menudo se deben a caídas o atropellos. La muerte por so- bredosis involuntaria de drogas o alcohol también es frecuente.

Otro peligro importante es la exposición a los elementos meteorológicos. En invierno hay un alto riesgo de congelación e hipotermia, mientras que en verano se pueden producir quemaduras solares graves e insolación.

$\mathrm{Al}$ contrario de la creencia popular, solo una pequeña proporción de individuos sin hogar son esquizofrénicos (6-13\%). Los trastornos afectivos son mucho más frecuentes (20-40\%). Los trastornos por consumo de alcohol son extremadamente frecuentes, con una prevalencia de $60 \%$ en los varones, cifra seis a siete veces mayor que en la población en general. En EE.UU., la prevalencia media de trastornos por consumo de drogas se ha estimado en $30 \%$. La cocaína (especialmente el crack) y la marijuana son las drogas ilícitas más consumidas por las personas sin hogar de Canadá.

Los adultos sin hogar tienen un alto consumo de atención sanitaria y a menudo reciben dicha atención en servicios de urgencias. Su ingreso en el hospital es hasta cinco veces más frecuente que en la población en general y las estancias son más prolongadas que las de otros pacientes con bajos ingresos. A menudo son trasladados a refugios, aunque no sea este el mejor medio para atender sus problemas. Las personas sin hogar se enfrentan a muchas barreras en su acceso a la atención sanitaria. En los EE.UU. uno de sus principales problemas es la falta de seguro de salud. Aunque en Canadá la cobertura del seguro de salud es universal, muchas personas sin hogar no pueden demostrar estar cubiertos, debido a pérdida o robo de sus documentos de identificación. Por otro lado, muchos de estos individuos no toman las medicaciones prescritas porque no pueden pagarlas. Además, hay otras barreras no relacionadas con el aseguramiento. La lucha diaria por la subsistencia puede hacerles percibir los problemas de salud como secundarios y muchas recomendaciones sobre reposo y cambios de dieta pueden ser imposibles de cumplir. El sistema de salud también fracasa frecuentemente en la provisión de tratamientos adecuados para las personas sin hogar con enfermedades mentales o problemas de abuso de drogas.

En resumen, la falta de hogar es un problema que afecta a un importante número de canadienses de todas las edades y que se asocia con una elevada morbilidad y mortalidad. El sistema de salud no cubre adecuadamente las necesidades de estos ciudadanos y son necesarias más investigaciones sobre la forma de cubrir dichas necesidades. La búsqueda de soluciones a largo plazo para el problema de la falta de hogar en sí misma sigue siendo una importante prioridad. (Hwang SW. Homelessness and health. CMAJ 2001;164:229-233). 
Aparatos de medición de la tensión arterial. Recomendaciones de la Sociedad Europea de Hipertensión

Hay un gran mercado para los aparatos de medición de la tensión arterial, no solo entre los médicos, sino también entre el público, una vez que las personas tienden cada vez más a medirse su propia tensión arterial. Para unos y otros, la exactitud debería ser un criterio fundamental a la hora de elegir el aparato de medición. Sin embargo, la exactitud de la mayoría de los aparatos todavía no ha sido investigada de forma independiente con los dos métodos de validación más utilizados: el de la Sociedad Británica de Hipertensión (British Hypertension Society; BHS) y el de la Asociación Estadounidense para el Avance del Instrumental Médico (US Association for the Advancement of Medical Instrumentation; AAMI). Por consiguiente, con el fin de orientar a los compradores, la Sociedad Europea de Hipertensión decidió examinar regularmente los aparatos de medición de la tensión. En este primer informe se incluyen los aparatos para los que existen datos publicados acerca de su validación con los métodos antes mencionados.

El criterio requerido por la BHS es que, en una escala de cuatro grados (de A a D, donde A representa la mayor concordancia y D la menor) los aparatos alcancen al menos el grado $B$, que implica que el porcentaje acumulado de mediciones de la tensión sistólica y de la tensión diastólica que difieran en $\leq 5, \leq 10$ y $\leq 15 \mathrm{~mm} \mathrm{Hg}$ con respecto a las mediciones efectuadas con el aparato estándar de mercurio sea, como mínimo, del 50, 75 y 90\%, respectivamente. A su vez, el criterio requerido por la AAMI es que las mediciones efectuadas con el aparato investigado y con el aparato estándar de mercurio no difieran en una media $>5 \mathrm{~mm} \mathrm{Hg}$ o una desviación típica $>8 \mathrm{~mm} \mathrm{Hg}$. Para recomendar un aparato se exigió que cumpliera ambos criterios tanto para la tensión sistólica como para la tensión diastólica.

Los aparatos se clasificaron en dos grandes grupos: esfigmomanómetros manuales (de mercurio o aneroides) y automáticos. Este último grupo se subdividió en aparatos de uso hospitalario, de uso ambulatorio, de uso comunitario y de uso personal. La información sobre los fabricantes aparece en la página de Internet de British Medical Journal.

Solo se recomienda el uso de 1 de los 2 esfigmomanómetros manuales probados (PyMah de mercurio); de 2 de los 5 esfigmomanómetros automáticos de uso hospitalario (Datascope Accutorr Plus y CAS Model 9010); de 5 de los 23 esfigmomanómetros automáticos de uso personal para medición de la tensión arterial en la arteria braquial (Omron HEM-705CP, Omron HEM-722C, Omron HEM-735C, Omron HEM-713C y Omron HEM-737
Intellisense); de ninguno de los 4 esfigmomanómetros automáticos de uso personal para medición de la tensión arterial en la arteria radial, y de 16 de los 24 esfigmomanómetros automáticos de uso ambulatorio (CH-DRUCK, Daypress 500, DIASYS Integra, ES-H531, Meditech ABPM-04, Profilomat, QuietTrak, Save 33 Model 2, Schiller BR-102, SpaceLabs 90202, SpaceLabs 90207, SpaceLabs 90217, TM-2420 Model 6, TM-2420 Model 7, TM-2421 y Takeda 2430), aunque algunos de estos solo se recomiendan en una de las dos modalidades (auscultatoria u oscilométrica), en determinados grupos de pacientes, como los ancianos o las embarazadas, o en algunas circunstancias, como el ejercicio o el reposo. La información existente acerca de la exactitud de los aparatos automáticos de uso comunitario, como los instalados en farmacias o supermercados, es muy escasa, y no se recomienda ninguno.

Aunque la exactitud de estos aparatos está recibiendo gran atención, las pruebas existentes no siempre son indiscutibles, debido al posible incumplimiento de los métodos recomendados, a la publicación de los datos de validación únicamente en forma de resumen o a las discrepancias entre los resultados de diferentes estudios de validación de un mismo aparato.

Hasta ahora solo se ha validado de forma independiente la exactitud de un pequeña parte de los numerosos aparatos existentes en el mercado, sobre todo de los destinados a uso personal y a uso hospitalario. Los esfigmomanómetros aneroides tienen el problema adicional de que se van haciendo más inexactos con el uso. Tampoco es fácil decidir cuál es la mejor forma de influir sobre los fabricantes para que modifiquen los aparatos que han demostrado ser inexactos y sobre los potenciales compradores para que reconozcan dicha inexactitud. La página de Internet de British Medical Journal proporcionará una actualización continua de la información sobre la validación y las recomendaciones acerca de los aparatos de medición de la tensión arterial. (O'Brien E, Waeber B, Parati G, Staessen J, Myers MG, on behalf of the European Society of Hypertension Working Group on Blood Pressure Monitoring. Blood pressure measuring devices: recommendations of the European Society of Hypertension. BMJ 2001;322:531-536).

\section{¿Qué entienden las mujeres por un "resultado normal del frotis cervical"?}

El no entender el riesgo residual inherente a la obtención de un resultado negativo en una prueba de tamizaje puede retrasar la búsqueda de atención ante la aparición de síntomas, producir inadaptación psicológica a la enfermedad o llevar incluso a 
emprender acciones legales. El programa de detección del cáncer cervical del Servicio Nacional de Salud del Reino Unido tiene por norma enviar a toda mujer con un resultado negativo en el frotis cervical una carta en la que se declara que el resultado de la prueba ha sido "normal", con el fin de evitar el término "negativo" para referirse a un hecho que la mujer considera "positivo", en el sentido de favorable. Además, se recomienda, pero no se exige, que en esa carta se declare que un resultado "normal" no significa que no haya riesgo de padecer o venir a padecer cáncer cervical, sino que dicho riesgo es bajo.

Con el fin de averiguar qué entienden las mujeres por un "resultado normal" y de investigar la eficacia de diferentes formas de presentar el riesgo residual inherente a la obtención de un resultado negativo, los autores realizaron un estudio experimental en el que participaron 1027 mujeres de 20 a 64 años de edad (media de $37,8 \pm 11,0$ ) que rellenaron cuestionarios en los que se les pedía que imaginaran que recientemente habían sido sometidas a un frotis de detección del cáncer cervical y que se les había notificado un "resultado normal". En realidad, 94\% de ellas habían sido sometidas a la prueba en el pasado y $21 \%$ habían tenido un resultado anormal. La tasa de falta de participación no se determinó formalmente, pero se estimó en menos de $5 \%$.

En un primer estudio participaron 153 mujeres a las que se les dijo que habían obtenido un "resultado normal" y otras 152 a las que, además, se les explicó que corrían un bajo riesgo de padecer o venir a padecer cáncer cervical en los próximos 5 años. En un segundo estudio participaron 722 mujeres que fueron asignadas a cuatro grupos. Uno recibió la misma información que el segundo grupo del estudio anterior, y los otros tres recibieron información adicional: a uno se le proporcionó una estimación numérica de su riesgo residual absoluto ( 1 en 5 000), a otro una estimación de su riesgo residual relativo en comparación con las mujeres no sometidas a la prueba (5 veces menor), y al tercero ambas estimaciones. Las mujeres rellenaron, sin ninguna ayuda, los cuestionarios en los que se les pedía que explicaran el significado del resultado, referido tanto al momento actual como a los 5 años siguientes. Ambas preguntas tenían seis respuestas opcionales: 1) Definitivamente no padezco (padeceré) cáncer cervical. 2) Es muy poco probable que padezca (venga a padecer) cáncer cervical. 3) Es poco probable que padezca (venga a padecer) cáncer cervical. 4) Es probable que padezca (venga a padecer) cáncer cervical. 5) Padezco (padeceré) cáncer cervical. 6) No sé. Se consideraron como interpretaciones correctas la segunda y la tercera. Además, se les preguntó su edad y estado civil, si se habían realizado alguna vez la prueba, y su nivel educacional, que solo era ligeramente inferior a la media de la población.

En el primer estudio comprendieron que había un riesgo residual de padecer cáncer cervical en la actualidad el $52 \%$ de las mujeres a las que únicamente se les comunicó que tenían un "resultado normal", frente al $70 \%$ de aquellas que también recibieron la explicación adicional sobre el significado del "resultado normal" (diferencia del 18\%; intervalo de confianza del $95 \%$ : $7 \%$ a $29 \% ; P=0,001)$. $\mathrm{La}$ diferencia también fue significativa $(P=0,02)$ con respecto al riesgo de padecer la enfermedad en los 5 años siguientes. El segundo estudio reveló que la información sobre el riesgo absoluto o relativo no mejora la comprensión con respecto a la obtenida con la información adicional del primer estudio. La edad, el estado civil y el haberse sometido anteriormente a la prueba no se relacionaron con la comprensión del resultado. El nivel educacional se relacionó directamente con la comprensión del significado del resultado referido al momento actual, pero no a los 5 años.

En resumen, este estudio revela que: 1) cerca de la mitad de las mujeres no entienden que un "resultado normal" del frotis cervical no significa que no haya un riesgo residual de padecer cáncer cervical en la actualidad o de venir a padecerlo en los próximos 5 años; 2) es posible mejorar la comprensión del resultado con una explicación sencilla de que, a pesar de la normalidad de la prueba, sigue existiendo un pequeño riesgo de padecer la enfermedad, y 3) dicha comprensión no mejora con la presentación de información numérica sobre el riesgo relativo ni absoluto. Teniendo en cuenta estos resultados, los autores creeen que el Servicio Nacional de Salud del Reino Unido debería cambiar la forma de comunicar la normalidad del frotis cervical, acompañando siempre la expresión "resultado normal" de una frase que especifique que esto no significa que no siga habiendo un pequeño riesgo de padecer cáncer cervical en la actualidad o de venir a padecerlo en el futuro. Estos resultados deberían ser confirmados en una muestra de mujeres realmente sometidas a la prueba con resultado normal, a pesar de que no es de esperar que fueran muy diferentes en esa situación. (Marteau TM, Senior V, Sasieni P. Women's understanding of a "normal smear test result": experimental questionnaire based study. BMJ 2001;322:526-528).

\section{Tendencias temporales del autismo y de la vacunación frente al sarampión, la parotiditis y la rubeola en California}

En los años 90, un grupo de investigadores británicos postuló que la vacunación frente al sa- 
rampión o la vacunación combinada frente al sarampión, la parotiditis y la rubeola (triple vírica) podrían ser una causa de autismo. Esta hipótesis ha suscitado grandes debates y a su favor se ha citado un informe de 1999 del Departamento Californiano de Servicios del Desarrollo (California Department of Developmental Services: CDDS) que mostraba un aumento del número de casos de autismo en la región a lo largo de las dos últimas décadas.

Sin embargo, el diagnóstico del autismo no siempre resulta fácil y los métodos de diagnóstico y la definición de los casos han sufrido cambios a lo largo del tiempo. Asimismo, hay que considerar la posibilidad de que la asociación temporal entre la vacunación triple vírica y el autismo se deba al azar, una vez que la vacuna se suele administrar entre los 12 y los 15 meses de edad y que la mediana de edad a la que los padres suelen notar por primera vez síntomas de autismo en sus hijos es a los 18 a 19 meses. Los autores de este estudio se propusieron determinar si existe una correlación entre las tendencias seculares de la vacunación triple vírica y del número de casos de autismo. Para ello examinaron los datos de California sobre la vacunación triple vírica en la infancia y los datos sobre los casos de autismo en niños nacidos entre 1980 y 1994, el último año para el que existen datos relativamente completos sobre los casos de autismo.

Los datos sobre la vacunación triple vírica de los niños californianos proceden de las encuestas anuales del Departamento de Servicios de Salud de California, en las que el método de muestreo se mantuvo prácticamente inalterado a lo largo de los años examinados. Se analizan dos medidas de la cobertura de la vacunación: las proporciones de niños vacunados con la triple vírica a los 17 y a los 24 meses de edad. La primera se utilizó por ser los 17 meses la edad que precede la edad mediana a la que los padres de los niños con autismo suelen notar los primeros síntomas, y la segunda para detectar una potencial tendencia secular al aumento de la cober- tura vacunal que no se hubiera detectado a los 17 meses de edad.

Entre 1980 y 1987 apenas hubo cambios en la cobertura vacunal con la triple vírica. En 1988 se produjo un moderado aumento, seguido de una nueva fase de meseta con escasos cambios en los 6 años siguientes. El aumento relativo de la cobertura a los 24 meses fue del 14\% entre $1980(72 \%)$ y 1994 $(82 \%)$.

Se observó una importante tendencia al aumento del número de casos de autismo a partir de 1985, bastante antes del aumento de la cobertura vacunal registrado en 1988. Además, dicha tendencia siguió manifestándose hasta 1994, lo cual significa que persistió mucho después de que la curva de cobertura vacunal volviera a estabilizarse tras el aumento registrado en 1988. Además, la tendencia al aumento del número de casos de autismo, de 176 en 1980 a 1182 en 1994 (aumento relativo de 572\%), fue muy superior al moderado aumento de la cobertura vacunal. Expresando los datos como tasas, el aumento relativo de los casos de autismo fue de 373\% (de 44 por 100000 nacidos en la cohorte de nacidos en 1980 a 208 por 100000 nacidos en la cohorte de nacidos en 1994).

La ausencia de correspondencia entre la tendencia de la cobertura vacunal triple vírica y la tendencia del número de casos de autismo no apoya la hipótesis de que el aumento de la vacunación triple vírica se asocie a la marcada tendencia secular al aumento del número de casos de autismo registrados por el CDDS. La misma ausencia de correlación se ha documentado también en el Reino Unido y Suecia. Los autores concluyen que las observaciones publicadas, basadas en datos empíricos, no indican que el aumento de la cobertura vacunal con la triple vírica en niños pequeños se asocie a la tendencia secular al aumento del número de casos de autismo. (Dales L, Hammer SJ, Smith NJ. Time trends in autism and in MMR immunization coverage in California. JAMA. 2001;285:1183-1185). 